\title{
High time-resolution analytical models for heat transfer through U- shaped ground heat exchangers
}

\section{Min Li}

TieMeng Sun

\author{
XueCheng Zhuo
}

\begin{abstract}
This paper reports a set of high time-resolution analytical models for heat transfer of GHEs, including a full scale line-source model for heat transfer outside U-shaped pipes and a quasi-3D heat transfer model for the circulating fluid inside U-shaped pipes. The full scale line-source model is a composite expression consisting of a composite medium line-source solution for short-term temperature responses, an infinite line-source solution for mid-term temperature responses, a finite line-source solution for long-term temperature responses, and a finite line-source solution for the thermal interaction between adjacent boreholes. The quasi-3D model for heat transfer inside U-pipes tackles the variation of the fluid temperature along the U-shaped channels. The fluid temperatures in the descending and ascending legs are derived as functions of time and borehole depth. The quasi-3D model, together with the full scale G-function, constitutes a complete theoretical model for heat transfer by ground heat exchangers, providing a computational-efficient approach to computer simulation, analysis, and optimum design of ground-coupled heat pump and ground heat storage systems.
\end{abstract}

\section{INTRODUCTION}

A great challenge in heat transfer analysis of ground heat exchangers (GHEs) is the involved diverse time and space scales (Li and Lai 2015; Li, et al. 2016). As shown in Li and Lai (2015) and Li et al. (2016), four space scales ranging from several centimeters to hundreds meters and eight time scales ranging from minutes to decades are probably involved in the heat transfer by borehole GHEs. A successful model should provide an accurate and efficient approach to calculating the diverse-scale thermal problem in the ground. From the perspective of accuracy, the heat transfer analysis should use a model of time resolution ranging from sub-hour to decades, corresponding to a space range from several centimeters to more than one hundred meters. From the efficient viewpoint, the analysis should tackle the complete spectrum of the broad time-length scales in an analytical way.

To meet the challenge in modeling GHEs, researchers have developed a vast number of analytical heat transfer models. Among them, conventional finite line-source models (FLS) appear to be the most suitable and efficient models for calculating the long-term temperature response in the ground (Claesson and Javed 2011), but they are unsuitable for modeling the short-term thermal response (Claesson and Javed 2011; Yang and Li 2014); the Infinite line-source solution (ILS) is only applicable to calculating the mid-term temperature response (Li, et al. 2016); and the infinite composite medium line-source model emerges as a valid model for calculating the short-term temperature response of GHEs (Li and Lai 2012; Li and Lai 2013; Yang and Li 2014), but it is invalid for the long-term process. An efficient analytical model that can address the entire time-space spectrum will be beneficial. Very few attempts 
have been made to develop such a model, however.

Claesson and Javed (2011) attempted to develop a heat transfer model covering time scales from minutes to decades; but they used the equilibrium-diameter assumption for their short-term model so that the used short- and long-term solutions are not guaranteed to blend. Li et al. (2014) developed a full scale line-source model by combining the composite-medium line-source solution and the conventional ILS and FLS solutions using the idea of matched asymptotic expansions. But the solution ignores the thermal interaction between adjacent boreholes and the vertical variation in fluid temperature along U-shaped tubes. The purpose of this paper is, based on our previous work, to extend the full scale line-source model to deal with these two thermal processes.

\section{HEAT TRANSFER MODEL}

Heat transfer by borehole GHEs spans a wide range of time and space scales; it is necessary to decompose the thermal process into two parts to simplify the analysis. We propose here decomposing the heat transfer of borehole GHEs by the outer wall of the U-shaped pipes: assume the heat transfer from the circulating fluid to the outer wall of the U-pipe to be a quasi-steady (or steady-flux) process and the heat transfer from the outer U-pipe wall to the ground to be a transient process. Further suppose that $T_{f 1}$ and $T_{f 2}$ are the fluid temperatures in the two legs of the U-pipe, which are functions of time $t$ and coordinate $z$ in the depth direction ( $z=0$ on the ground surface). The energy balance equations for $T_{f 1}$ and $T_{f 2}$ can be written as

$$
\begin{gathered}
c_{p} m \frac{\rrbracket T_{f 1}(t, z)}{\llbracket z}=\frac{T_{p 1}(t, z)-T_{f 1}(t, z)}{R_{p}} \\
-c_{p} m \frac{\prod T_{f 2}(t, z)}{\mathbb{\Upsilon} z}=\frac{T_{p 2}(t, z)-T_{f 2}(t, z)}{R_{p}}
\end{gathered}
$$

Eqs. (1) and (2) ignore the heat conduction in the fluids along the z-direction as a result of the small temperature gradient in this direction, but consider both the local horizontal thermal process through the U-shaped tube and the vertical variation of the fluid temperature along the U-shaped channel (Ma, et al. 2015). $R_{p}$ is the fluid-to-pipe thermal resistance of the $\mathrm{U}$-pipe (unit is $\mathrm{W} \cdot \mathrm{m} / \mathrm{K}$ ):

$$
R_{p}=\frac{1}{2 \pi k_{p}}\left(\ln \frac{r_{o}}{r_{i}}+\frac{k_{p}}{\alpha r_{i}}\right)
$$

The boundary conditions of Eqs. (1) and (2) are the temperature conditions on the ground surface $(z=0)$ and at the bottom of the borehole $(z=H)$, respectively: $T_{f 1}(t, 0)=T_{f, i n} . T_{f 2}(t, H)=T_{f 1}(t, H)$, where $T_{f, i n}$ is the inlet temperature of GHEs. Having these conditions, it is easy to solve Eqs. (1) and (2) analytically. And the final expression for the outlet temperature of GHEs, $T_{f, o u t}$, is (Ma, et al. 2015)

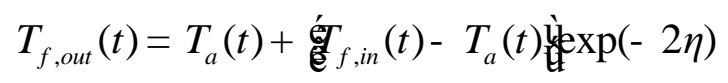

where $\eta$ is a dimensionless quantity having the similar implication as the number of transfer units (NTU):

$$
\eta=\frac{H}{c_{p} m R_{p}}
$$

Eq. (4) is applicable for GHEs using single U-tube and parallel-connected double U-tube. The similar idea is 
applicable to the case of series connected double U-tube (or W-shaped tube), and the outlet temperature response is (Ma, et al. 2015)

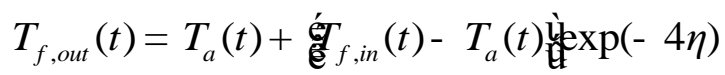

$T_{a}$ occurred in Eqs. (4) and (6) is the mean temperature of the outer walls of U-shaped pipes, which is unknown and needs to be determined. There are several approaches to determining $T_{a}$. The approach we used is by imposing a constant heat flux boundary condition on the outer walls because the heat flux can be easily determined by the heating and cooling loads and COP of the heat pump. By this assumption, $T_{a}$ can be determined using the concept of unitstep response function (i.e., G-function) as follows (Li et al. 2016; Li and Lai 2015):

$$
T_{a}=T_{0}+q_{l} G(t)
$$

$G$ function is the mean temperature of the outer wall of the U-shaped pipe due to a unit step change in the heat flux (i.e., $q_{l}=1$ in Eq. (7)). $G$ function used here has the same dimension as thermal resistance $R_{p}$ and their forms differ slightly from the dimensionless counterparts. It should be noted that Eqs. (4) - (7) embrace the natural way of the operation of GHEs: GHEs are driven by the inlet temperature of GHEs and the flow rate of the circulating fluid; that $T_{f, i n}$ and $m$, in conjunction with $T_{0}$, are used in Eqs. (4) - (7) as the boundary/driven conditions for computing $T_{f, o u t}$ reproduces the physical reality.

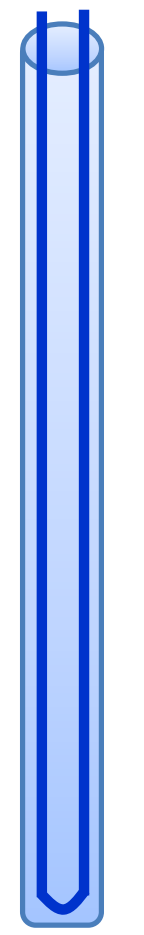

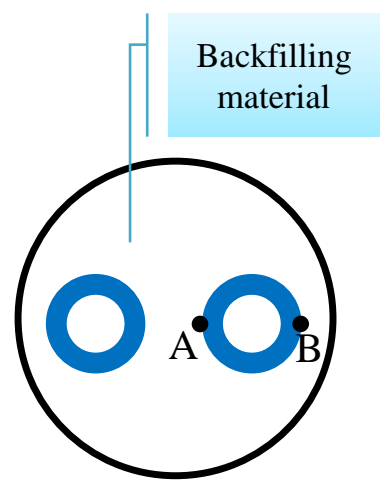

B) Borehole GHE with a single Ushaped tube. The coordinates of points $\mathrm{A}$ and $\mathrm{B}$ are $\left(D-r_{o}, 0\right)$ and $(D$ $+r_{o}, 0$ ), where $D$ is the half spacing between the pipe legs

A) Borehole GHE

Figure 1 The line-heat-source assumption used in the heat transfer model for U-shaped GHEs 
Following the idea, $G$ must be known to calculate $T_{a}$ and $T_{\text {fout }}$. This work uses the full scale line-source model for the $G$ function, which is a composite expression consisting of four temperature response functions as follows (Li, et al. 2014; Li, et al. 2016):

$$
G(t)=G_{i}+G_{o}-G_{m}+\Delta G
$$

Here, $G_{\mathrm{m}}$ is an infinite line-heat-source solution to the temperature response on the borehole wall (Carslaw and Jaeger 1959):

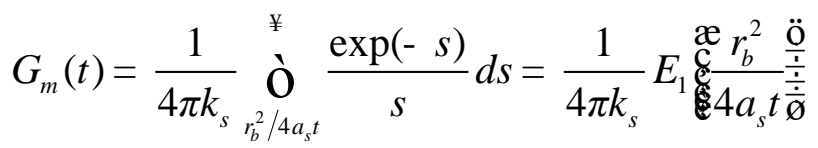

$G_{0}$ in Eq. (8) is a finite line-heat-source solution for the temperature of the borehole wall. According to Claesson and Javed (2011), the expression for the mean temperature of the borehole wall is:

$$
G_{o}(t)=\frac{1}{4 \pi k_{s}} \underset{1 / \sqrt{4 a_{s} t}}{\stackrel{\ddagger}{\mathbf{O}}} \exp \left(-r_{b}^{2} u^{2}\right) \frac{I\left(H u, H_{1} u\right)}{H u^{2}} d u
$$

where $I$ is a special function defined as follows:

$$
I\left(x_{1}, x_{2}\right)=2 \cdot \operatorname{ierf}\left(x_{1}\right)+2 \cdot \operatorname{ierf}\left(x_{1}+2 x_{2}\right)-\operatorname{ierf}\left(2 x_{1}+2 x_{2}\right)-\operatorname{ierf}\left(2 x_{2}\right)
$$

Here, $\operatorname{ierf}(x)$ denotes integral of the error function $\operatorname{erf}(x)$ :

$$
\operatorname{ierf}(x)=\int_{0}^{x} \operatorname{erf}(s) d s=x \cdot \operatorname{erf}(x)-\frac{1}{\sqrt{\pi}}\left[1-\exp \left(-x^{2}\right)\right]
$$

In Eq. (8), $G_{i}$ is a composite medium line-source solution to the average temperature of the outer wall of the Ushaped pipes. The key idea of the composite medium line-source solution is that the legs of U-shaped tubes (not the borehole) are modeled as lines of heat sources placed in a cylindrical composite medium. Thus it is possible to obtain the transient temperature field inside the borehole and the average temperature $T_{a}$. To simplify calculation, $T_{a}$ is approximated by the average temperature of points A and B as labeled in Fig. 1. Readers can find more details about this model in the references ( $\mathrm{Li}$ and Lai 2012; $\mathrm{Li}$ and Lai 2013). For single U-shaped tubes, $G_{\mathrm{i}}$ is

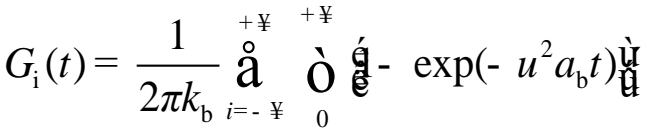

$$
\begin{aligned}
& \frac{J_{2 i}\left(u r_{A}\right)+J_{2 i}\left(u r_{B}\right)}{2} \frac{J_{2 i}\left(u r^{\prime}\right)(\varphi g-\psi f)}{u\left(\varphi^{2}+\psi^{2}\right)} d u
\end{aligned}
$$

For double U-shaped pipes, the expression of $G_{\mathrm{i}}$ is 


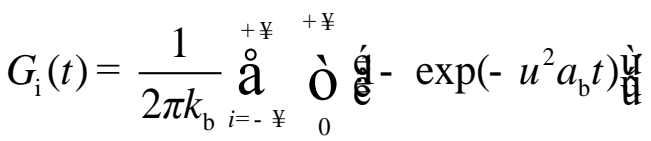

$$
\begin{aligned}
& \frac{J_{4 i}\left(u r_{A}\right)+J_{4 i}\left(u r_{B}\right)}{2} \frac{J_{4 i}\left(u r^{\prime}\right)(\varphi g-\psi f)}{u\left(\varphi^{2}+\psi^{2}\right)} d u
\end{aligned}
$$

where $r_{A}$ and $r_{B}$ are the radius coordinates of points $\mathrm{A}$ (e.g., $r_{A}=D-r_{0}$ ) and $\mathrm{B}$ (e.g., $r_{B}=D+r_{0}$ ) (see Fig. 1); functions $\varphi, \psi, f$, and $g$ are defined as

$$
\begin{aligned}
& \varphi=a k J_{n}\left(u r_{b}\right) J_{n}^{\prime}\left(a u r_{b}\right)-J_{n}^{\prime}\left(u r_{b}\right) J_{n}\left(a u r_{b}\right) \\
& \psi=a k J_{n}\left(u r_{b}\right) Y_{n}^{\prime}\left(a u r_{b}\right)-J_{n}^{\prime}\left(u r_{b}\right) Y_{n}\left(a u r_{b}\right) \\
& f=a k Y_{n}\left(u r_{b}\right) J_{n}^{\prime}\left(a u r_{b}\right)-Y_{n}^{\prime}\left(u r_{b}\right) J_{n}\left(a u r_{b}\right) \\
& g=a k Y_{n}\left(u r_{b}\right) Y_{n}^{\prime}\left(a u r_{b}\right)-Y_{n}^{\prime}\left(u r_{b}\right) Y_{n}\left(a u r_{b}\right)
\end{aligned}
$$

where $a$ and $k$ are dimensionless variable $k=k_{\mathrm{s}} / k_{\mathrm{b}}, a=\left(a_{\mathrm{b}} / a_{\mathrm{s}}\right)^{1 / 2} ; J_{n}$ and $Y_{n}$ denote the Bessel functions of the first kind and the second kind of order $n$, respectively; $J_{n}$ and $Y_{n}$ are the derivatives of $J_{n}$ and $Y_{n}$; the order $n$ is equal to $2 i$ and 4i, respectively, in Eqs. (13) and (14).

$\Delta G$ in Eq. (8) denotes the superimposed temperature due to the thermal interaction between adjacent boreholes. It is calculated by the finite line-source model:

$$
\Delta G(t)=\sum_{i=1}^{M-1} \frac{1}{4 \pi k_{s}} \int_{1 / \sqrt{4 a_{s} t}}^{\infty} \exp \left(-B_{i}^{2} u^{2}\right) \frac{I\left(H u, H_{1} u\right)}{H u^{2}} d u
$$

In summary, the $G$ function defined in Eq. (8) is a composite expression consisting of a finite line-source solution, an infinite line source solution, a composite medium line-source solution, and a finite line-source solution to the thermal interaction between boreholes. The underlying idea of this combined formula is the principle of matched asymptotic expansions (Li, et al. 2014; Li, et al. 2016). In fact, referring to Eq. (8), we can find that the short-term (high-frequency) temperature response is calculated only by $G_{i}$ if $G_{o}$ and $G_{m}$ cancel each other out within short time scales (e.g., times smaller $10 \mathrm{hr}$ ); the mid-term response is calculated by $G_{m}$ if $G_{0}$ can offset $G_{i}$ within this time period (from $10 \mathrm{hr}$ to several months); and the long-term response is determined only by $G_{0}$ if $G_{i}$ cancels out $G_{m}$ during large time periods (times larger several months). Therefore, it is essential for Eq. (8) that the $G$ functions (i.e., $G_{i}$, $G_{0}$, and $\left.G_{m}\right)$ must be derived from correct and complete theoretical models. Otherwise, they cannot cancel out each other in the overlapped time ranges. Since Eq. (8) synthesizes the advantages of short-, mid-, and long-term solutions, it is suitable for calculating GHE's temperature responses from several minutes to decades.

\section{RESULTS}

This section first provides a collection of graphs for $G$ functions generated by using the full-scale line-source solutions (i.e., Eqs. (8) - (16)). Compared to conventional G-functions, the new G-functions, which are anchored to the theoretically complete models, are more direct for use and, more importantly, are expected to be more reliable and accurate, being a new contribution to this field.

The new model integrates with the finite line-source model by the principle of superposition, making it feasible 
to calculate the thermal interaction between boreholes in a theoretical way. While this improvement is not new, this paper proposes a very fast but accurate way of calculating thermal interaction between large numbers of boreholes, a way that is based on systematic parameteric analysis. Our parameteric analysis reveals that the average temperataure reponse of a GHEs cluster can be well approximated by the average of the maximum and the minimum temperatures of the GHEs cluster (Fig. 2). Fig. 2 illustrates that the average of the minimum and the maxiumin temperatures agrees with the corresponding mean temperatures. The relative errors are only about $2 \%$. Additioinally, parametric analysis validates that this approximation is also applicable to other GHEs configurations, such as the linear and the L-shaped arrangements of GHEs.
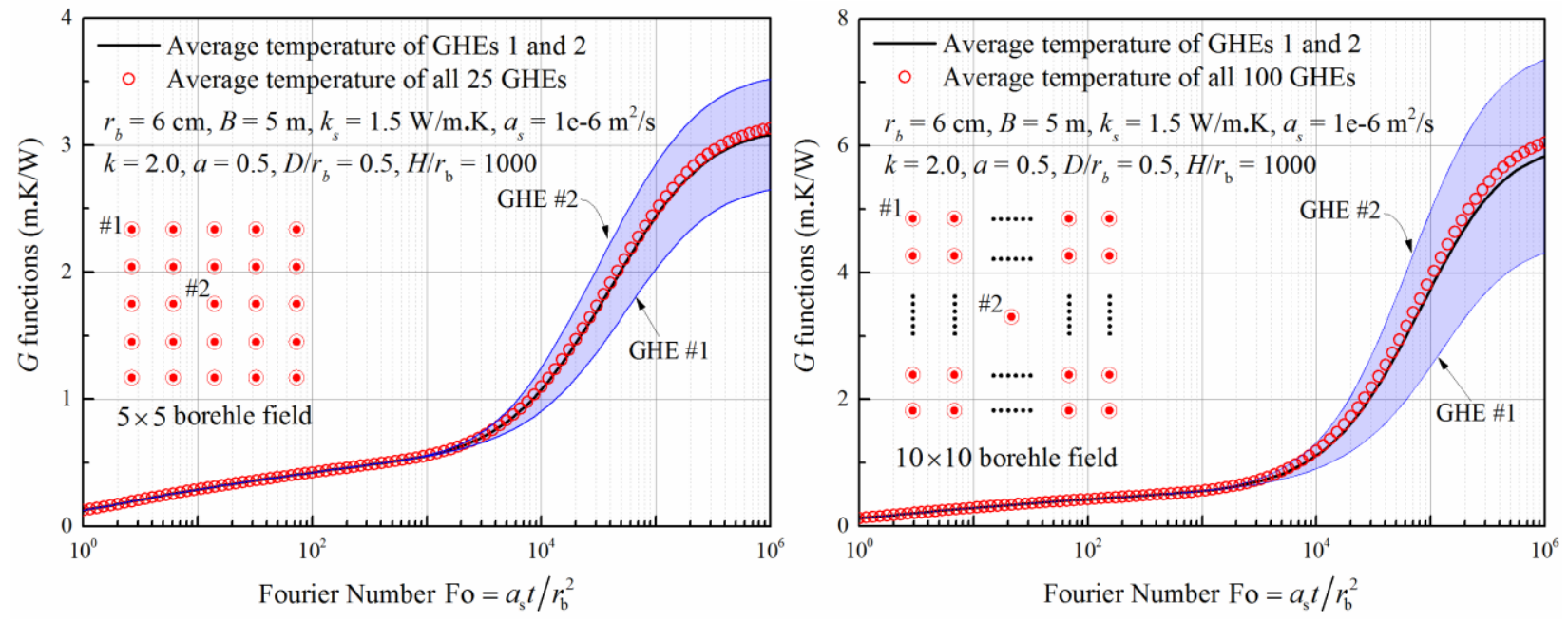

Figure 2 Temperature responses of $5 \times 5$ and $10 \times 10$ GHEs matrices: the maximum, the minimum, the average, and the approximated average temperature responses.
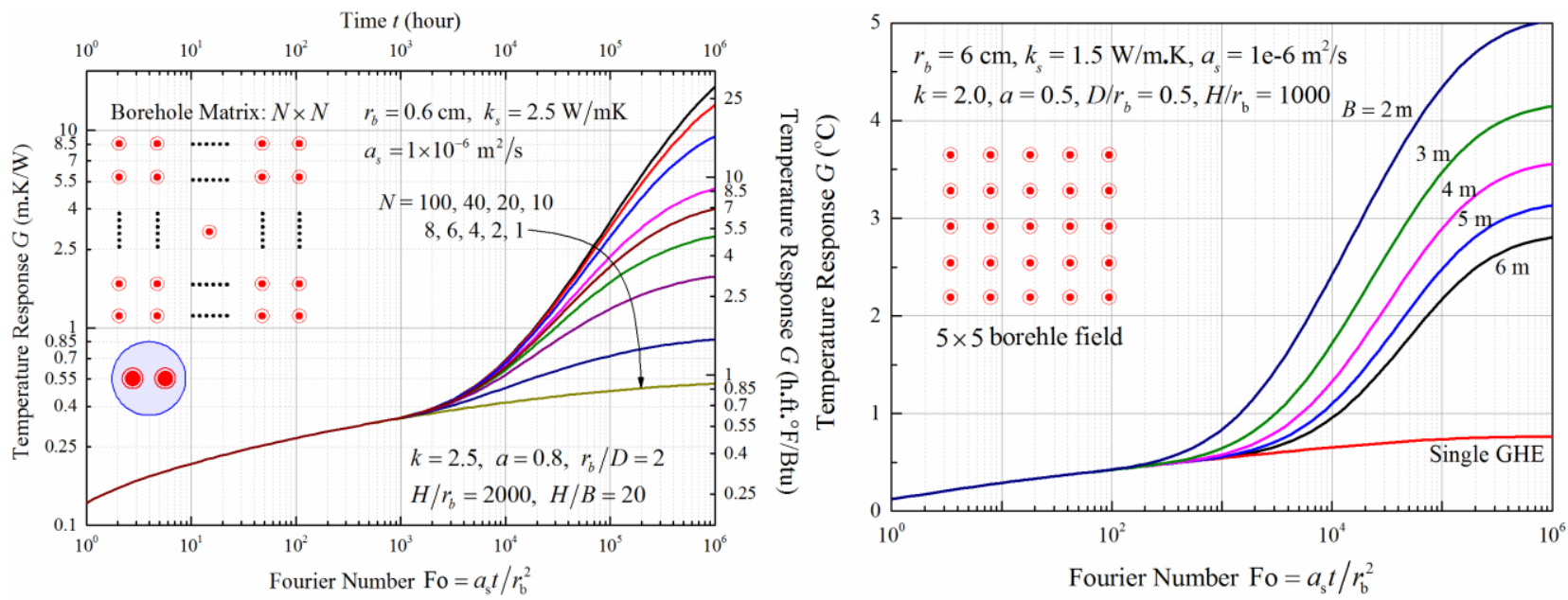

Figure 3 Temperature responses of GHEs matrices with different borehole numbers and spacing (from single borehole to 10000 boreholes). 
Fig. 3 shows general variations of the mean temperature responses of square GHEs clusters (i.e., $G$ functions). Similar charts have been reported by some researchrs (Eskilson 1987; Claesson and Javed 2011). Fig. 3A is complementary to previous charts by providing $G$ functions for extremely large GHE clusters (up to 10000 boreholes). It is important to remember that these curves are inadequate and incomplete for practical design because $G$ functions involve too many parameters to summarize in several 2D graphs. In practice, computerized approaches to $G$ functions would be more desirable and accurate.

Another improvement of the new model is the integration of the composite medium line-source model, which can accurately predict short-term temperature responses and avoid the complex computation of effective borehole thermal resistance $R_{b}$. As far as we know, the composite medium line-source solution is probably the best analytical solution (at least so far) to the short-term heat transfer of GHEs. This solution can yield transient temperature field inside boreholes, making the quasi-steady-state assumption unnecessary. Accordingly, $R_{b}$ used in the conventional models is not needed anymore.

The authors would like to draw more attention to the elimination of $R_{b}$ from the simulation. The authors tend to argue that $R_{b}$ is redundant for the thermal analysis of GHEs because not only it is more difficult to determine than $R_{p}$ but also it is a derivative of the assumption about the heat transfer inside boreholes. The computation of $R_{b}$ is difficult and uncertain, depending on the installation of U-tubes, the thermal properties of the ground and the backfilling material. Several theoretical formulas for $R_{b}$ exist, for example, the steady-state line-source and the firstorder multipole expressions for single U-tube GHEs (Hellström 1991). $R_{b}$ can be estimated from a thermal response test (Spitler and Gehlin 2015). No matter what method is used, however, the computation of $R_{b}$ is more complicated than that of $R_{p}$. In the new model, all the complications are lumped into the G-functions. A complete set of G-charts should be useful for engineering applications. Furthermore, the quasi-steady-state assumption is a seeming simplification to heat transfer analysis of GHEs, which introduces significant errors in the calculations of short-term or high-frequency temperature responses (Yang and Li 2014). This is the key reason why the study of short-term heat transfer has received a spate of interest recently (Lamarche and Beauchamp 2007; Javed and Claesson 2011; Li and Lai 2012; Lei, et al. 2015; Ruiz-Calvo, et al. 2015; Wei, et al. 2016). The composite medium line-source solution used here has been validated to be a viable solution to the problem of short-term thermal process ( $\mathrm{Li}$ and Lai 2013; Yang and Li 2014). Having this solution, it seems to us that the quasi-steady-state assumption is not needed anymore, neither is $R_{b}$.

\section{CONCLUSION}

This paper reports a set of theoretical solutions to heat transfer by borehole GHEs, which can predict temperature reponses of GHEs from sub-hour to decades. The solutions use the inlet temperature of GHEs, the flow rate of the circulating fluid, as well as the initial ground temperature as the boundary/driven conditions for computing the outlet temperature of GHEs, reproducing the physical reality of the operation of GHEs and providing a very direct way of GHEs simulation. Another outstanding feature of the new model is the ability to predict the transient heat conduction inside the boreholes. This ability enables the widely used effective borehole thermal resistance to be unnecessary, a derivative of the quasi-steady state assumption about the heat transfer inside boreholes.

\section{NOMENCLATURE}

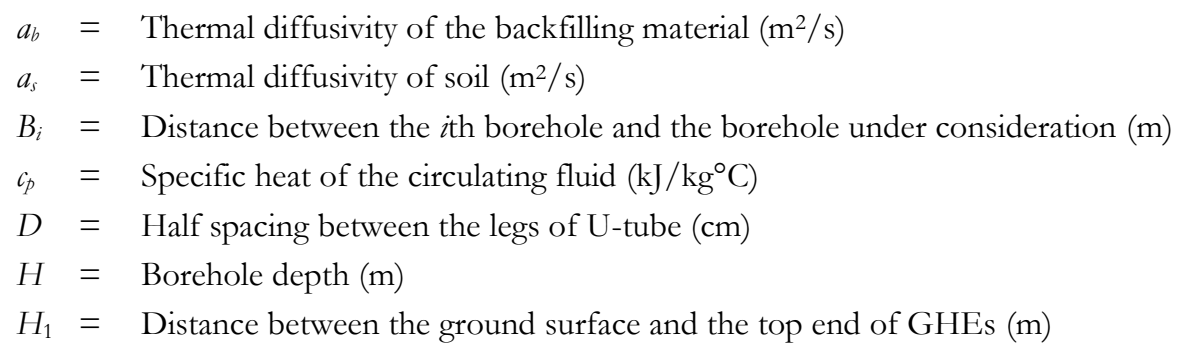




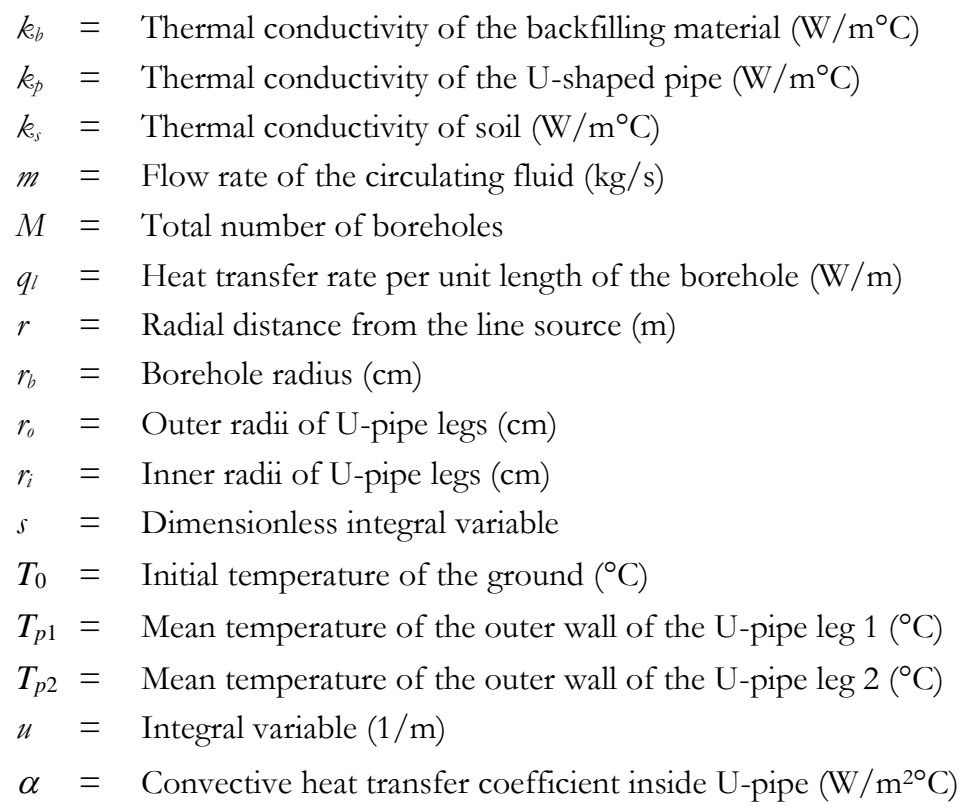

\section{REFERENCES}

Carslaw, H. S. and J. C. Jaeger. 1959. Conduction of heat in solids. Oxford, UK: Clarendon Press, 2nd ed.

Claesson, J. and S. Javed. 2011. An analytical method to calculate borehole fluid temperatures for time-scales from minutes to decades. ASHRAE Transactions 117(2): 279-288.

Eskilson, P. 1987. Thermal analysis of heat extraction boreholes. PhD thesis, University of Lund, Sweden.

Hellström, G. 1991. Ground heat storage - thermal analysis of duct storage systems I. Theory. Lund, Sweden: University of Lund.

Lamarche, L. and B. Beauchamp. 2007. New solutions for the short-time analysis of geothermal vertical boreholes. International Journal of Heat and Mass Transfer 50: 1408-1419.

Lei, F., P. F. Hu, N. Zhu and T. H. Wu. 2015. Periodic heat flux composite model for borehole heat exchanger and its application. Applied Energy 151: 132-142.

Li, M. and A. C. K. Lai. 2012. New temperature response functions ( $G$ functions) for pile and borehole ground heat exchangers based on composite-medium line-source theory. Energy 38: 255-263.

Li, M. and A. C. K. Lai. 2013. Analytical Model for short-time responses of borehole ground heat exchangers: Model development and validation. Applied Energy 104: 510-516.

Li M., P. Li, V. Chan and A. C. K. Lai. 2014. Full-scale temperature response function (G-function) for heat transfer by borehole ground heat exchangers (GHEs) from sub-hour to decades. Applied Energy 136: 197-205.

Li, M. and A. C. K. Lai. 2015. Review of analytical models for heat transfer by vertical ground heat exchangers (GHEs): A perspective of time and space scales. Applied Energy 151: 178-191.

Li, M., K. Zhu, and Z. H. Fang. 2016. Analytical methods for thermal analysis of vertical ground heat exchangers. In S. J. Rees Advances in Ground-Source Heat Pump Systems. Duxford: Woodhead Publishing.

Ma, W. W., M. Li, P. Li and A. C. K. Lai. 2015. New quasi-3D model for heat transfer in U-shaped GHEs (ground heat exchangers): Effective overall thermal resistance. Energy 90: 578 - 587.

Ruiz-Calvo, F., M. De Rosa, J. Acuña, J. M. Corberán and C. Montagud. 2015. Experimental validation of a short-term Borehole-toGround (B2G) dynamic model. Applied Energy 140: 210-223.

Spitler, J. D. and S. E. A. Gehlin. 2015. Thermal response testing for ground source heat pump systems-An historical review. Renewable and Sustainable Energy Reviews 50(0): 1125-1137.

Wei, J. P., L. Wang, L. Jia, K. Zhu and N. R. Diao. 2016. A new analytical model for short-time response of vertical ground heat exchangers using equivalent diameter method. Energy and Buildings 119: 13-19.

Yang, Y. and M. Li. 2014. Short-time performance of composite-medium line-source model for predicting responses of ground heat exchangers with single U-shaped tube. International Journal of Thermal Sciences 82: 130-137. 